\title{
Specificity of the Schrödinger equation
}

\author{
A. M. Cetto • L. de la Peña • \\ A. Valdés-Hernández
}

Received: 20 May 2015 / Accepted: 17 June 2015 / Published online: 21 July 2015

(C) Chapman University 2015

\begin{abstract}
As discussed in the first part of this paper, a key problem shared by some of the most well-known phenomenological derivations of Schrödinger-like equations is the (arbitrary) choice of the free parameters involved. Specifically, the (universal) constant $\hbar$ that makes the behavior of quantum systems so distinctive is normally introduced into the description by hand. This, however, does not need to be so. As shown in the present paper, the derivation provided by stochastic electrodynamics leaves no room for free parameters, the appearance of Planck's constant being determined univocally by the physics of the problem.
\end{abstract}

Keywords Schrödinger equation $\cdot$ Stochastic mechanics $\cdot$ Classical quantum analogs $\cdot$ Stochastic electrodynamics

\section{Introduction}

The Schrödinger equation is traditionally presented in textbooks either as a basic postulate or, alternatively, it is justified or 'derived' by making appeal to the wave nature of the solution $\psi$ of the equation and to de Broglie's relation $p=\hbar / \lambda$. In recent times, even simpler 'derivations' have been made available on the internet, spreading the idea that arriving at the Schrödinger equation is an easy matter. Then, there are the phenomenological derivations of Schrödinger-like equations in stochastic mechanics and the like, which seem to indicate that the most fundamental equation of quantum mechanics is simply one more wave equation to which a particular constraint is added. What is it, then, that makes the behavior of quantum systems so distinctive? What is their relation, if any, with those other (nonquantum) systems that obey a Schrödinger-like equation under certain conditions?

To find an answer to these important questions, it is necessary to clarify the real status of the various derivations. Given the scattered literature on the subject, a review on it would be most pertinent. Here, we offer a partial contribution to the topic, by focusing on a particular kind of approaches that have in common a stochastic element, and addressing the question of the uniqueness of the Schrödinger equation from a physical perspective as opposed

A. M. Cetto · L. de la Peña · A. Valdés-Hernández $(\bowtie)$

Instituto de Física, Universidad Nacional Autónoma de México, A.P. 20-364, Mexico, D.F., Mexico

e-mail: andreavh@fisica.unam.mx

A. M. Cetto

e-mail: ana@fisica.unam.mx

L. de la Peña

e-mail: luis@fisica.unam.mx 
to a merely formal one. Clearly, we do not pretend to cover all known proposals that have been made to arrive at the quantum description; in particular, among the interesting alternatives that escape the present discussion one may find those developed separately by Khrennikov [1-3] and by 't Hooft [4-6].

The paper starts by recalling the phenomenological derivation of Schrödinger-like equations in stochastic mechanics, which serves to stress the distinction between classical and quantum stochastic processes. This is followed by arguably the simplest derivation of all, which uses little more than the continuity equation and the factorization of the probability density in configuration space. The classical hydrodynamic analogies are then briefly discussed, particularly those that have been recently exhibited through the series of bouncing-drop experiments. The importance of the (arbitrary) choice of the free parameters involved in the various derivations of Schrödinger-like equations is highlighted. Finally, the derivation provided by stochastic electrodynamics is presented, showing that, contrary to the other approaches, it furnishes a physical explanation for the uniqueness of the Schrödinger equation, by univocally fixing the value of the parameters based on first principles.

\section{Stochastic (quantum) mechanics}

A variety of arguments have been given historically in support of the notion of a stochastic process underlying quantum mechanics. The more formal ones are based on the analogy first observed by Schrödinger [7,8] (and followed by Fürth [9]) between his equation and the diffusion equation, which are related with one another by analytical continuation into imaginary times. The seed planted by Schrödinger expanded much later into a fuller theory in terms of Bernstein processes [10-12]. A further argument that has been put forward is that if a quantum particle is considered to follow a stochastic process in configuration space, the resulting Hausdorff fractal dimension of such process is the same as that of a Brownian particle [13]. This fact is in itself interesting, although when the argument is transferred to a phase-space description the analogy breaks down. A number of additional reasons have been given in support of a stochastic approach to the quantum problem; for example, de Broglie [14] felt compelled to introduce it to make the particle switch at random from one guiding wave to another.

The first relatively accomplished stochastic theory of the quantum process was proposed by Féynes [15,16] on the basis of an ad hoc Lagrangian within a Brownian context. But perhaps the most widely known theory of this kind, based on a nondissipative Markov process, is stochastic mechanics, initiated by Nelson [17-19] and further developed by Guerra et al. [20,21], Davidson [22,23], and others. A common weakness of these works, at least during the earlier stages of their development, was the characterization of the underlying process as classical and of a Brownian nature. The need for a clear conceptual distinction between classical and quantum stochastic processes gave rise to a different branch of research, leading to the theory known as stochastic quantum mechanics [24-26]; this is the one that we will briefly recall. A somewhat different realist formulation of the stochastic approach to quantum mechanics has been developed more recently by Budiyono [27], following a Lagrangian schema instead of the Newtonian one presented here.

The theory uses a kinematics in configuration space that is sufficiently general to accommodate both classical and quantum processes. This is achieved by developing a coarse-grained description, with the concomitant appearance of two velocities: the diffusive (or osmotic) velocity $\boldsymbol{u}$ given by

$\boldsymbol{u}=D \frac{\nabla \rho}{\rho}=D \nabla \ln \rho$

where $\rho(\boldsymbol{x}, t)$ is the probability density and $D$ is the diffusion coefficient (which appears as a free parameter and is considered a scalar, for simplicity), and the systematic (or flow) velocity $\boldsymbol{v}$, which satisfies the continuity equation expressing the local conservation of particles,

$\frac{\partial \rho}{\partial t}+\nabla \cdot \rho \boldsymbol{v}=0$ 
Correspondingly, there appear four possible acceleration terms. The want of a specific model calls for the introduction of a couple of basic physical considerations for the construction of the dynamical law involving the four accelerations, namely their properties under time reversal and in the Newtonian (nonstochastic) limit. With $f=-\nabla V$, the external conservative force acting on the system, the general equation thus obtained $[25,26]$ reads

$m\left[\frac{\partial \boldsymbol{v}}{\partial t}+(\boldsymbol{v} \cdot \nabla) \boldsymbol{v}-\lambda(\boldsymbol{u} \cdot \nabla) \boldsymbol{u}-\lambda D \nabla^{2} \boldsymbol{u}\right]=\boldsymbol{f}$,

which can be integrated once, to obtain

$2 m D \frac{\partial S}{\partial t}+\frac{1}{2} m v^{2}-\lambda 2 m D^{2} \frac{\nabla^{2} \sqrt{\rho}}{\sqrt{\rho}}+V=0$,

where the dimensionless action function $S(\boldsymbol{x}, t)$ is such that

$v=2 D \nabla S$

and $\lambda$ is a free parameter, which may in principle have any real value. Equation (3) was first proposed by Nelson [17-19], with the specific value $\lambda=1$. The term in (4) that contains the Laplacian is known as the quantum potential. Equation (3) is obtained whenever the flow velocity $\boldsymbol{v}$ is irrotational (corresponding to a laminar flow) and can therefore be written as in Eq. (5). This restriction can be lifted, for example, when there is an external electromagnetic vector potential $\boldsymbol{A}$, in which case $\boldsymbol{v}=2 D \nabla S-(e / m c) \boldsymbol{A}$. The extra term appears eventually in the Schrödinger-like equation (see below), playing the usual role of the vector potential under minimal coupling.

Equations (4) and (2) form a couple of differential equations for the variables $\rho$ and $S$, which bear the dynamical information for an ensemble of particles subject to the conservative force $-\nabla V$. They can be decoupled by introducing the functions $\psi_{+}, \psi_{-}$such that

$\psi_{ \pm}=\sqrt{\rho} \exp \left( \pm \frac{S}{\sqrt{-\lambda}}\right)$

and

$\rho=\psi_{+} \psi_{-}$.

The velocities $\boldsymbol{v}$ and $\boldsymbol{u}$ rewrite then as

$\boldsymbol{v}=D \sqrt{-\lambda} \nabla \ln \frac{\psi_{+}}{\psi_{-}}, \quad \boldsymbol{u}=D \nabla \ln \psi_{+} \psi_{-}$.

This change of functions leads to the pair of uncoupled linear equations

$\mp i \gamma \frac{\partial \psi_{ \pm}}{\partial t}=-\frac{\gamma^{2}}{2 m} \nabla^{2} \psi_{ \pm}+V \psi_{ \pm}$,

where

$i \gamma \equiv 2 m D \sqrt{-\lambda}$.

Equations (9) apply to any system that is amenable to the present stochastic treatment, subject to the free (though nontrivial) choice of $D$ and $\lambda$, hence of $\gamma$. This exhibits at once the strength and the weakness of the procedure. For 
on the one hand, we have arrived at a Schrödinger-like description of the stochastic system; but on the other hand, the equation thus obtained is quite unspecific and the selection of the parameters seems rather arbitrary. A complete theory should allow for an unambiguous derivation of both $\lambda$ and the coefficients $D_{i j}$ (the elements of the diffusion tensor $\mathbb{D}$, which in the more general case is not necessarily constant nor diagonal as was assumed here), from first principles. This is beyond reach for the present phenomenological approach, due inter alia to the nonspecificity of the random field responsible for the stochasticity.

\subsection{Quantum vs classical stochastic processes}

Notice that in the integrated equations (9), the single free parameter is $\gamma$, i.e., the product $D \sqrt{-\lambda}$, not each factor separately. One may therefore consider that $D$ takes care of the scale, and take $\lambda= \pm 1$. This leads to essentially two different kinds of process, described by equations having a different mathematical structure, according to the sign of $\lambda$.

\subsubsection{The parabolic solution}

Take first $\lambda=-1$. In this case $i \gamma=2 m D$, Eq. (9) are parabolic, both $\psi_{+}$and $\psi_{-}$are real, and the process described by them is irreversible. This theory can be used to describe classical Markov processes, if due allowance is made for the unbalanced friction force, which can be introduced via an expression such as $\boldsymbol{f}_{\mathrm{f}}=-\beta \boldsymbol{v}_{e}$ with $\boldsymbol{v}_{e}=2 D\left(\nabla \psi_{+} / \psi_{+}\right)$, or the like (see, e.g., Ref. [28]). However, with the introduction of such a term, the theory ceases to be linear and it is then simpler to go back to the (linear) Fokker-Planck equation used traditionally to deal with Markov processes. Moreover, it happens that the values of both the friction parameter $\beta$ and the diffusion coefficient $D$ are problem-specific; a partial way out of this situation is the fluctuation-dissipation relation, when it is at hand. As is well known, the presence of the friction force, together with the incoherence of the noisy background, leads to purely noisy solutions for $t \rightarrow \infty$. With all these drawbacks, a procedure as the present one seems to be of limited practical value in this case.

\subsubsection{The hyperbolic solution}

With $\lambda=1$, according to (10), the parameter $\gamma$ becomes equal to $2 m D$, Eq. (6) gives

$\psi_{-}=\sqrt{\rho} \exp (i S)=\psi_{+}^{*}$,

and Eq. (9) becomes hyperbolic and each other's complex conjugate. The process is therefore reversible. The Schrödinger equation is obtained with the selection

$D=\frac{\hbar}{2 m}$

for the diffusion coefficient. It acquires the nature of a wavelike equation thanks to the factor $i$ in front of the first derivative with respect to time, which mimics a second-order time derivative-although giving rise to some bizarre wave phenomena; see, e.g., Refs. [29,30] for a discussion.

It should be stressed that the choice (12) is far from obvious or natural; there is no physical reason to assume that the diffusion tensor is diagonal and has a universal value, independent of the specific problem. Given the phenomenological character of the theory, this formula enters as an empirical selection, although there have been of course some attempts to justify it from fundamental considerations (see, e.g., Ref. [14]). As long as the task of identifying the noise source behind the assumed stochastic process is left open, that of deriving the values of $D$ and $\lambda$ remains open as well. 
It seems reasonable to ask at this point why the theory should predict an undulatory behavior, when the whole treatment has been made in terms of particles that follow (stochastic) trajectories. That such a stochastic mechanical formulation can reproduce the wavelike behavior of particles has been confirmed with the help of various numerical simulations. One such example is provided in the form of a fringe pattern obtained for a 'wave packet' of several thousand particles emerging from two Gaussian slits [31]. The result, obtained by numerical integration within stochastic mechanics, compares well, statistically speaking, with that obtained from a quantum mechanical calculation (J. Avendaño, Personal communication). Incidentally, an important observation made with this as with other numerical experiments [32-34] is that they demonstrate that each particle passes through just one slit.

\section{On Schrödinger-like equations}

From the above, it should be clear that deriving a Schrödinger-like equation is indeed a relatively easy matter. Yet, obtaining the Schrödinger equation is not. A true derivation of a quantum equation of motion requires more than arriving at a Schrödinger-like equation, it requires also deriving the value of the parameters involved, $\lambda$ and $D$. To make this point clear, suffice it to recall the following alternative way of arriving at the Schrödinger equation, based merely on general arguments of a statistical nature. The sole intention of this example is to elaborate on the general relationship between the Schrödinger equation and a simple stochastic description in configuration space.

The starting point is the continuity equation (2) for the density of particles [35]. Assuming again, for simplicity, that the flux is laminar we write the drift (systematic) velocity $\boldsymbol{v}$ in terms of a velocity potential (an 'action' $a S$ ) according to

$\boldsymbol{v}=\frac{a}{m} \nabla S$,

with the parameter $a$ selected so as to make $S$ a dimensionless function of $\boldsymbol{x}$ and $t$. A change of functions from $\rho, S$ to a complex pair $\psi, \psi^{*}$, defined as

$\psi=\sqrt{\rho} e^{i S}, \quad \psi^{*}=\sqrt{\rho} e^{-i S}$,

gives

$v=\frac{i a}{2 m} \nabla\left(\ln \psi^{*}-\ln \psi\right)$

The continuity equation becomes thus

$\psi^{*}\left(i \frac{\partial \psi}{\partial t}+\frac{a}{2 m} \nabla^{2} \psi\right)-\psi\left(-i \frac{\partial \psi^{*}}{\partial t}+\frac{a}{2 m} \nabla^{2} \psi^{*}\right)=0$

At this point, we introduce a separating real function $U(x, t)$ such that

$$
\begin{aligned}
\psi^{*}\left(i \frac{\partial \psi}{\partial t}+\frac{a}{2 m} \nabla^{2} \psi\right) & =\psi\left(-i \frac{\partial \psi^{*}}{\partial t}+\frac{a}{2 m} \nabla^{2} \psi^{*}\right) \\
& =U \psi \psi^{*} .
\end{aligned}
$$

As a result, Eq. (16) becomes separated into

$$
i \frac{\partial \psi}{\partial t}=-\frac{a}{2 m} \nabla^{2} \psi+U \psi
$$


and its complex conjugate. This procedure can be applied to any mechanical system obeying the continuity equation (with a laminar flow), and hence to classical or quantum particles alike. However, it remains to determine both the parameter $a$ and the function $U$, which may depend on $\boldsymbol{x}$, $t$ and even on $\psi$ and $\psi^{*}$. One procedure to determine $U$ is as follows (see Refs. [36,37] for other procedures).

We start by combining the expression (15) with Eq. (18), to obtain

$$
\frac{\partial \boldsymbol{v}}{\partial t}=\frac{a^{2}}{4 m^{2}} \nabla\left(\frac{1}{\psi^{*}} \nabla^{2} \psi^{*}+\frac{1}{\psi} \nabla^{2} \psi\right)-\frac{a}{m} \nabla U .
$$

On the other hand, $\nabla \ln \psi=(1 / 2) \nabla \ln \rho+i \nabla S$, whence

$$
\frac{\nabla^{2} \psi}{\psi}=\frac{\nabla^{2} \sqrt{\rho}}{\sqrt{\rho}}+i \nabla^{2} S+i \nabla S \cdot \frac{\nabla \rho}{\rho}-(\nabla S)^{2},
$$

which introduced into (19) leads to

$$
\frac{\partial \boldsymbol{v}}{\partial t}=\nabla\left(\frac{a}{2 m} \nabla \cdot \boldsymbol{u}+\frac{1}{2} \boldsymbol{u}^{2}\right)-\frac{1}{2} \nabla \boldsymbol{v}^{2}-\frac{a}{m} \nabla U,
$$

with

$\boldsymbol{u}=\frac{a}{2 m} \nabla \ln \rho$.

Using $\nabla \boldsymbol{v}^{2}=2(\boldsymbol{v} \cdot \nabla) \boldsymbol{v}$, we rewrite Eq. (20) in terms of the total time derivative along the trajectory $\mathrm{d} \boldsymbol{v} / \mathrm{d} t=$ $(\partial \boldsymbol{v} / \partial t)+(\boldsymbol{v} \cdot \nabla) \boldsymbol{v}$, as

$m \frac{\mathrm{d} \boldsymbol{v}}{\mathrm{d} t}=-\nabla\left(-\frac{a}{2} \nabla \cdot \boldsymbol{u}-\frac{1}{2} m \boldsymbol{u}^{2}\right)-a \nabla U$

On the other hand, Eq. (3) (with $D=a / 2 m$, in accordance with Eqs. (1) and (22)) can be recast in the form

$m \frac{\mathrm{d} \boldsymbol{v}}{\mathrm{d} t}=-\lambda \nabla\left(-\frac{a}{2} \nabla \cdot \boldsymbol{u}-\frac{1}{2} m \boldsymbol{u}^{2}\right)-\nabla V$

By combining the last two equations, we obtain

$$
(1-\lambda) \nabla\left(-\frac{a}{2} \nabla \cdot \boldsymbol{u}-\frac{1}{2} m \boldsymbol{u}^{2}\right)=\nabla(V-a U),
$$

which gives upon integration

$a U=V+(1-\lambda) V_{a}+h(t)$

with $h(t)$ an arbitrary function of time that can be taken as zero without loss of generality, and

$$
V_{a}=\left(\frac{1}{2} m \boldsymbol{u}^{2}+\frac{1}{2} a \nabla \cdot \boldsymbol{u}\right)=\frac{a^{2}}{2 m} \frac{\nabla^{2} \sqrt{\rho}}{\sqrt{\rho}} .
$$


We now introduce Eq. (26) into (18), thus obtaining

$i a \frac{\partial \psi}{\partial t}=-\frac{a^{2}}{2 m} \nabla^{2} \psi+V \psi+(1-\lambda) V_{a} \psi$

With $\lambda=1$, this becomes a Schrödinger-like equation,

$i a \frac{\partial \psi}{\partial t}=-\frac{a^{2}}{2 m} \nabla^{2} \psi+V \psi$

The value $\lambda=-1$, corresponding to the Brownian case as discussed above, leads instead to a (nonlinear) Schrödinger-like equation, with a total potential $V+2 V_{a}$. (The value $\lambda=0$ gives a net potential $V+V_{a}$, corresponding to a classical equation of motion devoid of stochasticity). Notice further that Eq. (25) is satisfied identically (for any $\boldsymbol{u}$ ) with the selection $\lambda=1, V=a U$.

Leaving aside the problem of justifying the selection $\lambda=1$ to arrive at (29), we observe that the parameter $a$ fixes the scale of the action $a S$, and is therefore in principle problem-dependent. Hence, the a priori identification of Eq. (29) with the Schrödinger equation containing a universal constant $a$ is not warranted. This observation explains the singular role played by Schrödinger's equation in quantum theory, and therein resides a specific feature of quantum systems. Whereas in the classical case the value of the action integrals is determined by the initial conditions (whence $a$ becomes highly arbitrary), in the quantum case this parameter becomes fixed in a more fundamental way: it is the initial conditions that are conditioned by the parameter, and determined so as to comply with an energy-balance condition, as will be shown in Sect. 5 .

Notwithstanding its importance, this discussion is frequently overlooked in the literature, characteristically in many published attempts to present variants of the above procedure as bona fide derivations of the Schrödinger equation from classical arguments.

An independent approach leading to the Schrödinger equation is provided by Reginatto [38,39], using information theory. For his derivation, the author introduces two basic assumptions: that one can associate a wave front with the motion of particles, and that the probability distribution for the position of particles should satisfy the principle of minimum Fisher information. While the first assumption introduces a physical element into the theory, and justifies the interpretation of the solution $\psi$ as a 'wave function', the association of Fisher information with the probability distribution incorporates an element of epistemic nature. The minimization procedure leads to the quantum mechanical equation, provided the minimization parameter-which in stochastic quantum mechanics represents the diffusion coefficient (multiplied by $\lambda=1$ ) - is assumed fixed and set equal to the appropriate value, which is precisely the one that introduces Planck's constant into the picture.

\section{Hydrodynamic analogies}

In the early phase of quantum mechanics, Madelung [40,41] demonstrated that the Schrödinger equation can be formally rewritten in hydrodynamic form. This so-called Madelung quantum hydrodynamics is usually considered as a precursor of the Bohmian mechanics [42,43], although the two theories are conceptually different. According to Madelung, the hydrodynamic-like velocity (5) appearing in the continuity equation (2) is not simply the particle velocity but an averaged quantity representing the flow of particles, hence $S$ is the (dimensionless) hydrodynamiclike velocity potential, rather than the mechanical action as in Bohm's theory. This formal hydrodynamic analogy has been useful to investigate qualitative properties of the nonlinear Schrödinger equation, particularly in the semiclassical limit, when the quantum pressure term (deriving from the quantum potential) can be considered small (see, e.g., Ref. [44]). Here, however, we are interested in a different kind of hydrodynamic analogy, namely the one observed in recent experiments exhibiting macroscopic quantum-like behavior. 


\subsection{Wavelike patterns}

Some 15 years ago, Couder and coworkers discovered that droplets bouncing on a vibrating fluid bath exhibit features previously thought to be exclusive of the quantum realm. These bouncers are macroscopic particles (millimetric drops of silicon oil) that can be made to dance over the surface of a vertically vibrated bath of the same fluid (which acts as a substratum), and to self-propel with constant speed. With this arrangement, a variety of phenomena having a striking similarity with the wavelike behavior of quantum particles have been observed, including single-particle diffraction, tunneling, quantized orbits and orbital level splitting [45-50].

A detailed theoretical analysis of the corresponding 'pilot-wave-hydrodynamics' has revealed that this macroscopic system can indeed be described, under very specific circumstances, i.e., within a limited range of values for the system parameters (specifically the drop size, driving acceleration and driving frequency), by a Schrödinger-like equation of the form (29). The parameter $a$ is then determined by the system parameters, which means that its value is problem-dependent, rather than universal as in Schrödinger's equation.

In recent years, Grössing et al. [51-53] have attempted to model a quantum as a nonequilibrium steady state maintained by a permanent throughput of energy, much in the spirit of the walking-droplet experiments. Specifically, they consider the particle as a bouncer whose oscillations are phase-locked with those of the energy-momentum reservoir of the surrounding vacuum, which they propose to identify with the zero-point radiation field. Introducing into the (otherwise classical) expression for the particle Lagrangian an additional kinetic energy term associated with the apparent temperature of the surroundings (emulating the quantum potential), they arrive at the (time-dependent) Schrödinger equation, introducing Planck's constant into the picture through the energy $E=\hbar \omega$ associated with each particle, considered as an elementary oscillator.

\section{Stochastic electrodynamics}

The discussion in previous sections points to at least two elements that in one way or another must be present in any process leading to the Schrödinger equation: the stochasticity, and the undulatory component. We have also seen that these two elements, though necessary, are nevertheless not sufficient to arrive univocally at Schrödinger's equation; at most they produce a Schrödinger-like equation with a parameter that is undetermined and may be problem-dependent. The fact that there are even classical, macroscopic systems that exhibit quantum-like features under specific constraints (and within a limited range of system parameters) may be an indication that classical and quantum systems are perhaps not so distant from one another as usually considered; however, the Schrödinger equation, with Planck's constant $\hbar$ as a universal parameter, is unique, and it is specific of quantum mechanics. What is the physical origin of this uniqueness?

The answer to this question has been central to the theory of stochastic electrodynamics [54,55]. By considering that the (otherwise classical) particle is embedded in the fluctuating zero-point radiation field (corresponding to the temperature-independent term in Planck's blackbody radiation formula, see Eq. (33) below), this theory contains from the outset a physical element that provides in principle for both the stochasticity and the undulatory component mentioned above (and for the appearance of $\hbar$ ). It is important to realize that these ingredients are internal to the theory (rather than additional postulates). But rather than being innate to the particle, stochasticity and wavelike behavior appear as properties acquired by it through the permanent action of this specific kind of reservoir, which is the background field of energy $\hbar \omega / 2$ per normal mode. The question, now, is whether this physical element determines the quantum solution unequivocally.

The procedure leading to the Schrödinger equation for this problem starts with the equation of motion for the particle subject to an external conservative force $\boldsymbol{f}(\boldsymbol{x})$ and embedded in the random field [55-59]. A statistical treatment usual in the theory of stochastic processes $[60,61]$ leads in the Markovian limit to the Fokker-Planck equation for the probability density $Q(\boldsymbol{x}, \boldsymbol{p}, t)$, 


$$
\begin{aligned}
\frac{\partial Q}{\partial t} & +\frac{1}{m} \nabla_{\boldsymbol{x}} \cdot(\boldsymbol{p} Q)+\nabla_{\boldsymbol{p}} \cdot[(\boldsymbol{f}+m \tau \dddot{\boldsymbol{x}}) Q] \\
& =\nabla_{\boldsymbol{p}} \cdot\left(\mathbb{D}^{p p} \cdot \nabla_{\boldsymbol{p}}+\mathbb{D}^{p x} \cdot \nabla_{\boldsymbol{x}}\right) Q .
\end{aligned}
$$

In the first row we have, in addition to the external force $f$, the radiation-reaction force $m \tau \dddot{\boldsymbol{x}}\left(\tau=2 e^{2} / 3 m c^{3}\right)$; in the second row, we have two diffusive terms due to the action of the random field, with the components of the diffusion tensors given by

$D_{i j}^{p p}=e^{2} \int_{-\infty}^{t} \mathrm{~d} t^{\prime} \varphi\left(t-t^{\prime}\right) \frac{\partial p_{j}(t)}{\partial p_{i}\left(t^{\prime}\right)}$,

$D_{i j}^{p x}=e^{2} \int_{-\infty}^{t} \mathrm{~d} t^{\prime} \varphi\left(t-t^{\prime}\right) \frac{\partial x_{j}(t)}{\partial p_{i}\left(t^{\prime}\right)}$,

where

$\varphi\left(t-t^{\prime}\right)=\frac{4 \pi}{3} \int_{0}^{\infty} \rho_{0}(\omega) \cos \omega\left(t-t^{\prime}\right) \mathrm{d} \omega$

expresses the autocorrelation function of the zero-point field, of spectral energy density

$\rho_{0}(\omega)=\frac{\hbar \omega^{3}}{2 \pi^{2} c^{3}}$,

and $p_{i}\left(t^{\prime}\right)$ evolves deterministically towards $p(t)$. The entire system is assumed to approach eventually a reversible regime, as a result of the combined action of the (dissipative) radiation reaction and the (fluctuating) zero-point field force. In this regime, the average power lost by the particle by radiation is compensated by the average power gained from the field, and the residual effects of these terms become negligible. In this so-called radiationless approximation, the reduction of the description to the configuration space leads from Eq. (30) to a couple of equations that can be written in the form

$$
\begin{aligned}
& \frac{\partial \rho}{\partial t}+\boldsymbol{\nabla} \cdot(\boldsymbol{v} \rho)=0, \\
& m \frac{\partial}{\partial t}(\boldsymbol{v} \rho)+m \boldsymbol{\nabla} \cdot(\boldsymbol{v} \boldsymbol{v} \rho) \\
& \quad-\frac{\eta^{2}}{m}\left[\rho \boldsymbol{\nabla} \boldsymbol{\nabla}^{2} \ln \rho+(\boldsymbol{\nabla} \rho) \cdot \boldsymbol{\nabla}(\boldsymbol{\nabla} \ln \rho)\right]-\boldsymbol{f} \rho=0 .
\end{aligned}
$$

Notice that Eq. (35) contains a free parameter $\eta$ with units of action, which arises from a change of variables carried out in the transition to configuration space. The same parameter appears in the expressions for the local average momentum and its square average,

$\langle\boldsymbol{p}\rangle_{\boldsymbol{x}}=m \boldsymbol{v}=-i \eta \nabla \ln \frac{q(\boldsymbol{x}, t)}{q^{*}(\boldsymbol{x}, t)}$,

$\left\langle\boldsymbol{p}^{2}\right\rangle_{\boldsymbol{x}}=-\eta^{2} \nabla^{2} \ln \rho+\langle\boldsymbol{p}\rangle_{\boldsymbol{x}}^{2}$,

where $q^{*} q=\rho$.

By their form, Eqs. (34) and (35) resemble the corresponding pair of equations of kinetic theory [62], with the stress tensor replaced by the term proportional to $\eta^{2}$ in (35). To carry further this analogy (which goes back to Ref. $[40,41])$, one would have to treat this term as a kind of stress- - which is somewhat artificial, as no real fluid is 
supporting such stress. In the present context, the term proportional to $\eta^{2}$ has a clear meaning, since according to (37) it comes directly from the momentum fluctuations transcribed to configuration space. An important property of this term is its nonlocal nature, manifested through its dependence on the probability distribution in the entire configuration space, $\rho(\boldsymbol{x})$. Upon integration of (35), this term acquires precisely the form of the quantum potential

$V_{Q}=-\frac{\hbar^{2}}{8 m}\left[\frac{2}{\rho} \nabla^{2} \rho-\frac{1}{\rho^{2}}(\nabla \rho)^{2}\right]=-\frac{\hbar^{2}}{2 m} \frac{\nabla^{2} \sqrt{\rho}}{\sqrt{\rho}}$,

representing actually a contribution to the kinetic energy.

Upon integrating and combining Eqs. (34) and (35), one gets [55]

$\left[-2 i \eta \frac{\partial}{\partial t}+\frac{1}{2 m}(-2 i \eta \nabla)^{2}+V\right] q=h(t) q$,

with $h$ a real, but otherwise arbitrary function of time. In terms of the function

$\psi(\boldsymbol{x}, t)=q(\boldsymbol{x}, t) e^{-\frac{i}{2 \eta} \int^{t} h\left(t^{\prime}\right) \mathrm{d} t^{\prime}}$,

one finally arrives at

$-\frac{2 \eta^{2}}{m} \nabla^{2} \psi+V \psi=2 i \eta \frac{\partial \psi}{\partial t}$

with $\psi^{*} \psi=q^{*} q=\rho(\boldsymbol{x}, t)$. Without loss of generality, one may take $h(t)=0$, and hence $\psi(\boldsymbol{x}, t)=q(\boldsymbol{x}, t)$.

Equation (41) has precisely the form of the Schrödinger equation for the probability amplitude $\psi$, in terms of the parameter $\eta$. It is therefore again a Schrödinger-like equation, like (29), as long as $\eta$ remains undetermined. But we do have a physical basis to determine it, because we know that for the system to admit stationary solutions, it must have reached a state of energy balance with the background field. The explicit expression for this condition of energy balance is obtained by multiplying the Fokker-Planck equation (30) by $\boldsymbol{p}^{2}$ and integrating over the entire space, which gives

$\frac{\mathrm{d}}{\mathrm{d} t}\langle H\rangle=\tau\langle\boldsymbol{p} \cdot \dddot{\boldsymbol{x}}\rangle+\frac{1}{m}\left\langle\operatorname{Tr} \mathbb{D}^{p p}\right\rangle$,

where $H=\frac{1}{2 m} \boldsymbol{p}^{2}+V(\boldsymbol{x})$ is the particle Hamiltonian, and the components of $\mathbb{D}^{p p}$ are given in (31). Applied to the ground state (in which the mechanical system is in equilibrium with the zero-point field), the energy-balance condition reads

$\tau\langle\boldsymbol{p} \cdot \dddot{\boldsymbol{x}}\rangle_{0}=-\frac{1}{m}\left\langle\operatorname{Tr} \mathbb{D}^{p p}\right\rangle_{0}$

Using the stationary solutions of Eq. (41) (still in terms of $\eta$ ) to calculate both sides of this equation, one obtains in terms of the matrix elements $\boldsymbol{x}_{0 k}=\int \psi_{0}^{*} \boldsymbol{x} \psi_{k} \mathrm{~d}^{3} x$ and $\boldsymbol{p}_{0 k}=i m \omega_{0 k} \boldsymbol{x}_{0 k}$,

$\tau\langle\boldsymbol{p} \cdot \dddot{\boldsymbol{x}}\rangle_{0}=-m \tau \sum_{k} \omega_{0 k}^{4}\left|\boldsymbol{x}_{0 k}\right|^{2}$ 
and

$$
-\frac{1}{m}\left\langle\operatorname{Tr} \mathbb{D}^{p p}\right\rangle_{0}=-\frac{\hbar m \tau}{2 \eta} \sum_{k} \omega_{0 k}^{4}\left|\boldsymbol{x}_{0 k}\right|^{2} .
$$

Note that Planck's constant appears here as a footprint of the zero-point field. From equating these two results, it follows that for energy balance to hold, $\eta$ must have the value

$\eta=\frac{\hbar}{2}$

The general equation (41) becomes then precisely Schrödinger's equation,

$i \hbar \frac{\partial \psi}{\partial t}=-\frac{\hbar^{2}}{2 m} \nabla^{2} \psi+V \psi$

Therefore, the energy-balance condition (43) determines Schrödinger's equation univocally. From this perspective, the Schrödinger equation describes more than just the particle; it describes the particle in interaction with the field that has taken it to the time-reversible regime and has impressed upon it the characteristic fluctuating and wavelike properties.

The fact that $\eta$ does not depend on the specific problem (e.g., on the external potential $V$ ) but only on the zeropoint field of fixed intensity conveys to the Schrödinger equation its recognized universal validity. In addition to introducing Planck's constant into the picture and furnishing the right value for $\eta$, Eq. (43) confirms the spectrum $\rho \sim \omega^{3}$ as the single one that leads to equilibrium with the ground state of the mechanical system, by guaranteeing that both expressions (44) and (45) have the same algebraic structure. This means that the energy-balance condition is satisfied not only globally, but term by term (for every $k$ ), i.e., that a situation of detailed energy balance between particle and field is reached.

A comment is in place here regarding the nature of the zero-point field. This field is considered by many as quantum for the simple reason that it contains Planck's constant. However, it should be clear that we are not dealing with a quantized field, but with a solution of the (classical) Maxwell equations that does not go to zero at zero temperature. This is perfectly allowed by classical physics, and is in agreement with Wien's law for the mean energy of the field oscillators as a function of frequency and temperature (with $f$ an unspecified function in the general case), namely

$U(\omega, T)=\omega f(T / \omega)$,

as can be shown through a thermodynamic analysis [63]. At zero temperature, the energy per mode of frequency $\omega$ of the oscillators as allowed by Wien's law is $\mathcal{E}_{0}=U(T=0)=A \omega$, with $A=f(0)$ a universal constant with units of action [55,64-66]. The solution $A \neq 0$ represents a departure from the classical realm (it is contrary to energy equipartition) and leads to a zero-point field spectral energy density $\rho_{0}=A \omega^{3} / \pi^{2} c^{3}$. In Eq. (33), we have set the value $A=\hbar / 2$ to establish contact with the experimental value of the universal constant. In fact, we could have carried out all the derivations in this section writing $A$ instead of $\hbar / 2$. The result would have been the appearance of $A$ in the Schrödinger equation, and the relation between $\rho_{0}$ and this universal constant would have indicated that, as concluded above, the ZPF is the ultimate responsible for the specificity of the Schrödinger equation.

\section{Conclusions}

A main conclusion to be drawn from the above discussion refers to the degree of arbitrariness implicit in usual derivations (whether through stochastic mechanics, Fisher information theory, or some other stochastic or hydro- 
dynamic model) leading to a Schrödinger-like equation with a free parameter. The universality of this parameter (as well as its correct numerical value $\hbar$ ) should be a prediction of any theory that aims to explain (or reproduce) quantum mechanics from first principles. The fact that stochastic electrodynamics satisfies this demand strongly suggests that the zero-point radiation field plays a fundamental role in determining the quantum behavior of matter.

Acknowledgments The authors are grateful for valuable suggestions from an unknown referee. Financial support from DGAPAUNAM through projects PAPIIT IN106412 and IN112714 is acknowledged.

\section{References}

1. Khrennikov, A.: Beyond Quantum. Pan Stanford Publishing, Singapore (2014)

2. Khrennikov, A.: Born's formula from statistical mechanics of classical fields and theory of hitting times. Physica A 393, 207 (2014)

3. Khrennikov, A., Nilsson, B., Nordebo, S.: Quantum rule for detection probability from Brownian motion in the space of classical fields. Theor. Math. Phys. 174, 298 (2013)

4. 't Hooft, G.: The obstinate reductionist's point of view on the laws of physics. In: Proceedings of Europa-Vision und Wirklichkeit, Europäisches Forum Alpbach, p. 296. E. Busek Verlag, Ö sterreich (2001)

5. 't Hooft, G.: In: Elitzur, A., Dolev, S., Kolenda, N. (eds.) Quo Vadis Quantum Mechanics? Philadelphia (2002)

6. 't Hooft, G.: A mathematical theory for deterministic quantum mechanics. J. Phys. Conf. Ser 67, 012015 (2007)

7. Schrödinger, E.: Sitzungsber. Preuss. Akad. Wissen. Berlin, Phys. Math. Klasse, p. 144 (1931)

8. Schrödinger, E.: Sur la théorie relativiste de l'électron et l'interpretation de la mécanique quantique. Ann. Inst. Henri Poincaré 2 , 269 (1932)

9. Fürth, R.: Über einige Beziehungen zwischen klassischer Statistik and Quantenmechanik. Z. Phys. 81, 143 (1933)

10. Jamison, B.: Reciprocal processes. Z. Wahrscheinlichkeitstheorie verw. Gebiete 30, 65 (1974)

11. Zambrini, J.-C.: Stochastic mechanics according to E. Schrödinger. Phys. Rev. A 33, 1532 (1986)

12. Garbaczewski, P.: Physical significance of the Nelson-Newton laws. Phys. Lett. A 172, 208 (1993)

13. Abbott, L.F., Wise, M.B.: Dimension of a quantum-mechanical path. Am. J. Phys. 49, 37 (1981)

14. de Broglie, L.: Le mouvement brownien d'une particule dans son onde. C. R. Acad. Sci. Paris B 264, 1041 (1967)

15. Fényes, I.: A deduction of Schrödinger equation. Acta Bolyaina 1, 5 (1946)

16. Fényes, I.: Eine wahrscheinlichkeitstheoretische Begründung und Interpretation der Quantenmechanik. Z. Phys. 132, 81 (1952)

17. Nelson, E.: Derivation of the Schrödinger equation from Newtonian mechanics. Phys. Rev. 150, 1079 (1966)

18. Nelson, E.: Dynamical Theories of Brownian Motion, Mathematical Notes. Princeton University Press, NJ (1967)

19. Nelson, E.: Quantum Fluctuations. Princeton University Press, NJ (1985)

20. Guerra, F., Marra, R.: Origin of the quantum observable operator algebra in the frame of stochastic mechanics. Phys. Rev. D 28, $1916(1983)$

21. Guerra, F.: Stochastic variational principles and quantum mechanics. Ann. Inst. Henri Poincaré 49, 314 (1988)

22. Davidson, M.: A classical realization of quantum mechanics. Found. Phys. 8, 481 (1978)

23. Davidson, M.: Foundations of probability and physics. In: Adenier, G., Fuchs, C.A., Khrennikov, A.Y. (eds.) AIP Conference Proceedings, vol. 889. AIP, NY (2007)

24. de la Peña, L.: New Formulation of Stochastic Theory and Quantum Mechanics. J. Math. Phys. 10, 1620 (1969)

25. de la Peña, L., Cetto, A.M.: Stochastic theory for classical and quantum mechanical systems. Found. Phys 5, 355 (1975)

26. de la Peña, L., Cetto, A.M.: Does quantum mechanics accept a stochastic support? Found. Phys. 12, 1017 (1982)

27. Budiyono, A.: Objective uncertainty relation with classical background in a statistical model. Physica A 392(43), 307 (2013)

28. Cetto, A.M.: Investigaciones sobre una teoría estocástica de la mecánica cuántica, Ph. D. thesis. UNAM, Mexico (1972)

29. Ballentine, L.E.: Quantum Mechanics. Prentice Hall, NJ (1990)

30. Ballentine, L.E.: Quantum Mechanics. A Modern Development. World Scientific, Singapore (1998)

31. McClendon, M., Rabitz, H.: Numerical simulations in stochastic mechanics. Phys. Rev. A 37, 3479 (1988)

32. Webb, G.F.: Event-based interpretation of Schrödinger's equation for the two-slit experiment. Int. J. Theor. Phys. 50, 3571 (2011)

33. Michielsen, K., Yuan, S., Zhao, S., Jin, F., De Raedt, H.: Coexistence of full which-path information and interference in Wheeler's delayed-choice experiment with photons. Physica E 42, 348 (2010)

34. Michielsen, K., Jin, F., Delina, M., De Raedt, H.: Event-by-event simulation of nonclassical effects in two-photon interference experiments. Physica Scripta T 151, 014005 (2012)

35. de la Peña, L.: A simple derivation of the Schrödinger equation from the theory of Markov processes. Phys. Lett. A 24, 603 (1967)

36. Kracklauer, A.F.: An intuitive paradigm for quantum mechanics. Physics Essays 5, 226 (1992)

37. de la Peña, L., Cetto, A.M.: in Courants, amers, é cueils en microphysique. Fondation Louis de Broglie, Paris (1993)

38. Reginatto, M.: Derivation of the equations of nonrelativistic quantum mechanics using the principle of minimum Fisher information. Phys. Rev. A 58, 1775 (1998)

39. Klein, U.: The statistical origins of quantum mechanics. Phys. Res. Int. 2010, 808424 (2010)

40. Madelung, E.: Eine anschauliche Deutung der Gleichung von Schrödinger. Naturwissenschaften 14, 1004 (1926) 
41. Madelung, E.: Quantentheorie in hydrodynamischer form. Z. Phys. 40, 322 (1927)

42. Bohm, D.: A suggested interpretation of the quantum theory in terms of "hidden" variables. I. Phys. Rev. 85(166), 180 (1952)

43. Bohm, D., Hiley, D.J.: The Undivided Universe. An Ontological Interpretation of Quantum Theory. Routledge, London (1995)

44. Carles, R., Danchin, R., Saut, J.-C.: Madelung. Gross-Pitaevskii and Korteweg. Nonlinearity 25, 2843 (2012)

45. Couder, Y., Protière, S., Fort, E., Boudaoud, A.: Dynamical phenomena: walking and orbiting droplets. Nature 437, 208 (2005)

46. Couder, Y., Fort, E.: Single-particle diffraction and interference at a macroscopic scale. Phy. Rev. Lett. 97, 154101 (2006)

47. Oza, A., Rosales, R., Bush, J.: A trajectory equation for walking droplets: hydrodynamic pilot-wave theory. J. Fluid Mech. 737, $552(2013)$

48. Wind-Willassen, O., Moláček, J., Harris, D.M., Bush, J.W.: Exotic states of bouncing and walking droplets. Phys. Fluids 25, 082002 (2013)

49. Bush, J.W.: Pilot-wave hydrodynamics. Ann. Rev. Fluid Mech. 49, 269 (2015)

50. Brady, R., Anderson, R.: Analogue Physics. A Student's Guide to Waves in an Ideal Fluid. Cambridge University Computer Center, Cambridge (2013)

51. Grössing, G.: The vacuum fluctuation theorem: exact Schrödinger equation via nonequilibrium thermodynamics. Phys. Lett. A 372, 4556 (2008)

52. Grössing, G.: On the thermodynamic origin of the quantum potential. Physica A 388, 811 (2009)

53. Grössing, G.: Mesa Pascasio, J., Schwabl, H.: A classical explanation of quantization. Found. Phys. 41, 1437 (2011)

54. de la Peña, L., Cetto, A.M.: The Quantum Dice. An Introduction to Stochastic Electrodynamics. Kluwer, Dordrecht (1996)

55. de la Peña, L., Cetto, A.M., Valdés-Hernández, A.: The Emerging Quantum. The Physics Behind Quantum Mechanics. Springer, Switzerland (2015)

56. Cetto, A.M., de la Peña, L., Valdés-Herná ndez, A.: J. Quantization as an emergent phenomenon due to matter-zeropoint field interaction. Phys. Conf. Ser. 361, 012013 (2012)

57. Cetto, A.M., de la Peña, L.: Valdés-Herná ndez, A.: Quantum behavior derived as an essentially stochastic phenomenon. Physica Scripta T 151, 014008 (2012)

58. de la Peña, L., Valdés-Hernández, A., Cetto, A.M., França, H.M.: Genesis of quantum nonlocality. Phys. Lett. A 375, 1720 (2011)

59. de la Peña, L., Cetto, A.M.: Derivation of quantum mechanics from stochastic electrodynamics. J. Math. Phys. 18, 1612 (1977)

60. Papoulis, A.: Probability, Random Variables, and Stochastic Processes, Chap. 16. McGraw-Hill, Boston (1991)

61. Jacobs, K.: Stochastic Processes for Physicists. Understanding Noisy Systems. Cambridge University Press, Cambridge (2010)

62. Fujita, S., Godoy, S.V.: Mathematical Physics. Wiley-VCH, Weinheim (2010)

63. Boyer, T.H.: Random electrodynamics: The theory of classical electrodynamics with classical electromagnetic zero-point radiation. Phys. Rev. D 11, 790 (1975)

64. Boyer, T.H.: Thermodynamics of the Harmonic Oscillator: Wien's Displacement Law and the Planck Spectrum. Am. J. Phys. 71, $866(2003)$

65. Cole, D.C.: Derivation of the classical electromagnetic zero-point radiation spectrum via a classical thermodynamic operation involving van der Waals forces. Phys. Rev. A 42, 1847 (1990)

66. de la Peña, L., Valdés-Hernández, A., Cetto, A.M.: Statistical consequences of the zero-point energy of the harmonic oscillator. Am. J. Phys. 76(10), 947 (2008) 